\title{
Spatial autocorrelation analysis of 13 leading malignant neoplasms in Taiwan: a comparison between the 1995-1998 and 2005-2008 periods
}

\author{
Pui-Jen Tsai $^{{ }^{*}}$, Cheng-Hwang Perng ${ }^{2}$ \\ ${ }^{1}$ Center for General Education, Aletheia University, New Taipei, Taiwan; ${ }^{*}$ Corresponding Author: puijentsai@gmail.com \\ ${ }^{2}$ Department of Statistics and Actuarial Science, Aletheia University, New Taipei, Taiwan.
}

Received 23 September 2011; revised 10 November 2011; accepted 21 November 2011.

\begin{abstract}
Spatial autocorrelation methodologies, including Global Moran's I and Local Indicators of Spatial Association statistic (LISA), were used to describe and map spatial clusters of 13 leading malignant neoplasms in Taiwan. A logistic regression fit model was also used to identify similar characteristics over time. Two time periods (1995-1998 and 2005-2008) were compared in an attempt to formulate common spatio-temporal risks. Spatial cluster patterns were identified using local spatial autocorrelation analysis. We found a significant spatiotemporal variation between the leading malignant neoplasms and well-documented spatial risk factors. For instance, in Taiwan, cancer of the oral cavity in males was found to be clustered in locations in central Taiwan, with distinct differences between the two time periods. Stomach cancer morbidity clustered in aboriginal townships, where the prevalence of Helicobacter pylori is high and even quite marked differences between the two time periods were found. A method which combines LISA statistics and logistic regression is an effective tool for the detection of space-time patterns with discontinuous data. Spatio-temporal mapping comparison helps to clarify issues such as the spatial aspects of both two time periods for leading malignant neoplasms. This helps planners to assess spatio-temporal risk factors, and to ascertain what would be the most advantageous types of health care policies for the planning and implementation of health care services. These issues can greatly affect the performance and effectiveness of health care services and also provide a clear outline for helping us to better understand the results in depth.
\end{abstract}

Keywords: Spatial Autocorrelation Analysis; Global Moran's I Statistic; Local Indicators of Spatial Association Statistic; Logistic Regression; Malignant Neoplasm; Taiwan

\section{INTRODUCTION}

Spatial analytical techniques and models can identify spatial anomalies in the epidemiology of diseases, identify "hot spots" and locate spatio-temporal patterns. Cluster mapping clarifies issues of internal and external correlations, while logistic regression is a useful approach for the differentiation of spatial distribution patterns over time. Common spatial techniques for health research include: disease mapping, clustering techniques, diffusion studies, identification of risk factors through comparisons, and regression analyses [1]. All of these methods are useful when assessing risk factors. They also facilitate the planning of health care policies and support the implementation of effective health care services.

Cuzick and Edwards (1990) [2] proposed three general methodologies for the detection of clustering. Spatial autocorrelation statistics, such as Moran's $I$ [3-6] and Geary's $C$ [3-5] are global methods used to estimate the overall degree of spatial autocorrelation in a dataset. However, the possibility of spatial heterogeneity suggests that the estimated degree of autocorrelation may vary significantly. Local spatial autocorrelation statistics provide estimates disaggregated to the unit level, allowing the assessment of dependency relationships in different areas. LISA detect local spatial autocorrelation in aggregated data by dividing Moran's I statistic into contributions for each area within a study region. These indicators can detect clusters of similar or dissimilar disease frequency values around a given observation [7]. Unlike Moran's I statistic, which measures the correlation between attribute values in adjacent areas, the Gi(d) 
local statistic is an indicator of local clustering that measures the "concentration" of a spatially distributed attribute variable [8,9].

The analysis of spatio-temporal change is a major concern in geographical research. Analytical approaches include: the Knox test [10], Mantel's $Z$ statistic [11], the Jacquez $k$ nearest neighbor test [12], Kulldorff's spatial scan statistic [13-15] and Bayesian spatial scan statistic [16]. Herein, we are primarily interested in detecting clusters that emerge over time, and our goal is to detect emerging clusters as early as possible. For example, in the public health domain, our goal is to detect emerging clusters of disease indicative of naturally occurring disease outbreaks (such as influenza), bioterrorist attacks (such as anthrax release), or environmental hazards (such as a radiation leak). Clearly, the early detection of such clusters would contribute to a more rapid response, leading to lives being saved.

Cancer is one chronic disease with a multi-stage progression. Many studies examine cancer incidence at different times, under different environmental exposures and in different ethnic groups. Cancer incidence changes over time for people of different ages, which may be due to variations in lifestyle, changing environmental exposure, etc. Cancer incidence also varies in different geographic locations [17-20]. Again, this may have various explanations with environmental impact being a strong possibility.

The detection of spatio-temporal clustering generally requires continuous data. Discontinuous data, with different durations of disease surveillance at the same location, present a challenge. This study focuses on the use of a set of discontinuous data to detect changes in spatiotemporal clustering. We propose herein a method for ascertaining spatial clustering associated with the 13 leading malignant neoplasms, based on medical-care data collected by the Taiwan National Health Insurance and Taiwan Cancer Registry agencies. To test this approach, we have compared local clusters between two periods (1995-1998 and 2005-2008) looking for similarities. We have also investigated potential spatial risks that could contribute to these health care events, redefining epidemiologic and spatially referenced data.

\section{MATERIALS AND METHODS}

\subsection{Study Area}

The study area included the main island of Taiwan (excluding all surrounding islets) which, in the year 2000, comprised more than 22 million inhabitants living in an area of $36,000 \mathrm{~km}^{2}$. A total of 350 local administrative government areas, including five main urban areas, two secondary urban areas, 162 rural townships, and 54 aboriginal townships on the plain and in mountainous regions, were assessed (Figure 1). According to a 2002 Ministry of Interior report, urban areas are classified as regions having at least one metropolitan centre, and they can include neighboring cities and townships that share socio-economic activities. Main urban areas are defined as those with a population larger than one million, specifically, Taipei-Keelung, Kaohsiung, Taichung-Changhua, Jhongli-Taoyuan and Tainan. Secondary urban areas are defined as those with a residential population ranging from 0.3 to 1 million (e.g. Hsinchu and Chiayi).

\subsection{Data Collection and Management}

The Taiwan National Health Insurance (NHI) program was initiated in 1995. The coverage rate of the program increased from $92.4 \%$ in 1995 to more than $96.2 \%$ in 2000 , increasing to $98 \%$ after the inclusion of those active in the military forces in 2001. Once the NHI medical care data were properly collected and analyzed, a complete picture of population behaviors according to disease could be used for reference in the calculation of prevalence and incidence of various diseases.

At the beginning of 2004, NHI data that was available relative to medical care, such as the leading causes of death, were reclassified and reprocessed in relation to smaller units or areas (for example, precincts or townships rather than the country as a whole). In addition, regional data from the statistical analysis system (SAS) program are now announced publicly by the NHI in

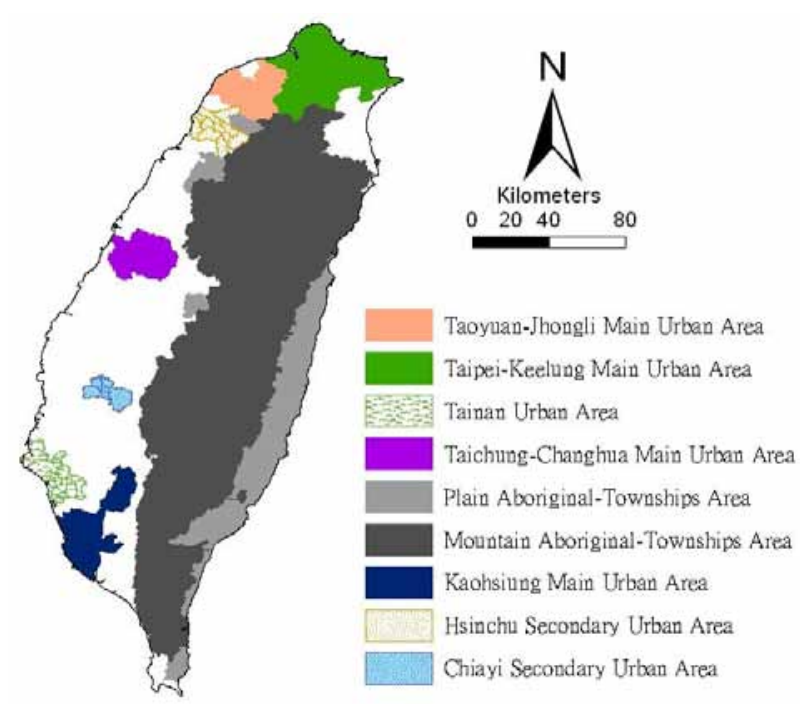

Figure 1. Map of urban areas and aboriginal townships in the study area. Map of the study area divided into 350 administrative districts including seven urban areas and an integrated area of 54 plains and mountain aboriginal townships.

regular annual reports (for example, NHI, 2005-2008 [21-24]). These reports provide an accurate and reliable 
data source for the investigation of health care issues in Taiwan.

Data were collected from contractual medical-care institutions, where the NHI covers the costs of prescription medicines and treatment at outpatient clinics. Such facilities accumulate detailed databases on medical costs for inpatient care. The number of outpatient cases were classified in relation to disease codes, as defined in the 1975 edition of "The International Classification of Diseases, 9th Revision, Clinical Modification” (ICD 9 CM). Patients suffering from diseases that were difficult to classify into a given code or had mismatched ID numbers were not included in the final statistical data set. Disease codes were classified according to gender and age. Cases with the same ID numbers, but which exhibited different diseases, were counted as different instances.

Medical care data obtained from the 2005-2008 NHI reports were examined, and the morbidity rates of the 13 leading causes of death were calculated. Disease classifications (according to the ICD 9 CM) included the following (indicated within parentheses): trachea, bronchus, and lung cancer (ICD 162); liver and intrahepatic bile ducts cancer (ICD 155); colon and rectum cancer (ICD 153, 154); stomach cancer (ICD 151); oral cavity cancer (ICD 140, 141, 143-146, 148, 149); oesophagus cancer (ICD 150); pancreas cancer (ICD 157); non-Hodgkin's lymphoma (ICD 200, 202, 203); gallbladder and extrahepatic bile ducts cancer (ICD 156); leukaemia (ICD 204-208); female breast cancer (ICD 174); cervix uteri cancer (ICD 179, 180); and prostate cancer (ICD 185).

Demographic information was provided by the Ministry of Interior [25]. The smallest administrative units coded for examination of the various diseases cases or health care events were precincts and townships. Ageadjusted standard morbidity rates, adjusted using the Segi ("world") population in 1976 as the standard [26], were then calculated providing results giving the leading causes of death for males and females in each township.

During the period from 1995 to 1998, data on ageadjusted malignancies by precinct and township were obtained from the Atlas of Cancer Mortality and Incidence in Taiwan, officially published by the Bureau of Health Promotion, Department of Health [27].

\subsection{Statistics}

The global Moran's I spatial autocorrelation was used to assess the correlation among neighbouring observations and to identify patterns and levels of spatial clustering in neighbouring districts [28]. The Moran's I statistic, similar to the Pearson correlation coefficient [29], was calculated by the following formula:

$$
I=\frac{N}{S_{O}} \sum_{i} \sum_{j} w_{i j} \frac{\left(x_{i}-\bar{x}\right)\left(x_{j}-\bar{x}\right)}{\sum_{i}\left(x_{i}-\bar{x}\right)^{2}}
$$

where $N$ is the number of districts, $w_{i j}$ the element in the spatial weight matrix corresponding to the observation pair $i, j$ and $x_{i}$ and $x_{j}$ observations for the areas $i$ and $j$ with the mean $\bar{x}$ and:

$$
S_{O}=\sum_{i} \sum_{j} w_{i j}
$$

Since the weights were row-standardized $\left(\sum w_{i j}=1\right)$, the first step in the spatial autocorrelation analysis was to construct a spatial weight matrix that contained information about the neighbourhood structure for each location. Adjacency was defined as immediately neighboring administrative districts, including the district itself. Non-neighbouring administrative districts were assigned the weight of zero.

Spatial contiguity for polygons is defined as the property of sharing a common boundary or vertex. Contiguity analysis is an important method for assessing unusual features in connectivity distribution $[4,30]$. The Queen's measure of contiguity can be utilized to make up for spatial contiguity by incorporating both the Rook and Bishop relationships into a single measure [30]. The administrative districts considered in this study were highly irregular in both shape and size. Tsai et al. (2009) demonstrated that the most appropriate method is the first order queen polygon contiguity method for quantifying the spatial weights matrix for the analysis of connectivity. Based on this approach, the spatial weight/ connectivity matrices were determined and utilized in conjunction with the global Moran's I statistic and following LISA calculations [6].

Moran's $I$ values may range from -1 (dispersed) to +1 (clustered). A Moran's $I$ value of 0 suggests complete spatial randomness. A random permutation procedure recalculates a statistic many times by reshuffling the data values among the map units to generate a reference distribution. The obtained calculated statistic based on the observed spatial pattern is then compared to this reference distribution and a pseudo significance level (pseudo $p$-value) computed. To verify that the value of Moran's I was significantly different from the expected value, we applied a Monte Carlo randomisation test with 999 permutations to achieve highly significant values. Data values were reassigned among the $\mathrm{N}$ locations, providing a randomised distribution against which one may judge the observed value. If the observed value of $I$ was within the tails of this distribution, there was significant spatial autocorrelation in the data, a pseudo $p$-value smaller than 0.05 , and the assumption of independence among the observations could be rejected [31].

LISA statistic provides information related to the lo- 
cation of spatial clusters and outliers and the types of spatial correlation. Local statistics are important because the magnitude of spatial autocorrelation is not necessarily uniform over the study area [7,32]. LISA allowed us to divide the study area into small locations, thus enabling the assessment of significant local spatial clustering around an individual location. In addition to the degree of spatial clustering, detailed variations of clustering in the locally defined geo-space were identified as well as the locations of the spatial clusters. The local version of Moran's $I$ at location $i$ is given by:

$$
I_{i}=\frac{\left(x_{i}-\bar{x}\right)}{\frac{1}{n} \sum_{i}\left(x_{i}-\bar{x}\right)^{2}} \sum_{j} w_{i j}\left(x_{j}-\bar{x}\right)
$$

where $n$ indicates the total number of locations (350 townships used in the years 1995-1998 and 349 townships in 2005-2009); $x_{i}$ denotes the value of the variable of interest, $X$, at location $I ; x_{j}$ denotes the observation at neighboring locations $j$; and $\bar{X}$ is the sample average of $X . w_{i j}$ is the spatial weight matrix, which defines spatial interaction across study regions. In general, $w_{i j}=1$ if location $i$ and location $j$ are neighboring, (share a common boundary); otherwise, $w_{i j}=0$. In this study, spatial contiguity was assessed as the first order queen's contiguity which defines spatial neighbors as those areas with shared borders and vertexes.

Significance was tested by comparison to a reference distribution obtained by random permutations [7]. This analysis used 999 permutations to determine differences between spatial units. A positive value for the local Moran's $I$ index $\left(I_{i}\right)$ indicates that a feature has neighboring features with similarly high or low attribute values and is therefore part of a cluster. A negative value for $\left(I_{i}\right)$ indicates that a feature has neighboring features with dissimilar values; this feature is an outlier. In either instance, the $p$-value for the feature must be small enough for the cluster or outlier to be considered statistically significant. LISA enables distinguishment between a statistically significant (0.05 level) cluster of high values $(\mathrm{HH})$, a cluster of low values (LL), an outlier in which a high value is surrounded primarily by low values (HL), and an outlier in which a low value is surrounded primarily by high values ( $\mathrm{LH})$. In addition to the value of a $\mathrm{z}$-score larger than +1.96 , the outcomes are defined as clusters with both $\mathrm{HH}$ and LL. In the case of a value of a z-score less than -1.96 , the outlier is considered as clusters with (HL) and (LH). We consider that outliers may not be stablily and precisely displayed the outcomes of spatiotemporal pattern comparison, because it is difficult to distinguish between outliers how strength with or without disease risks. Therefore, only hot and cold spots are mapped on local Moran's maps.
In addition to mapping, similarities between spatial distribution patterns for the two periods (1995-1998 and 2005-2008) were determined using logistic regression analysis. The binary response indicates whether there is significant autocorrelation between administrative districts or areas. The correlation is better (higher) if the value of the z-score of the local Moran's I statistic is larger than +1.96 (clusters with hot spots and cold spots), otherwise it is deemed to be low. The model is expressed as:

$$
\log \left(\frac{\operatorname{Pr}(\text { Higher correlation })}{\operatorname{Pr}(\text { Lower correlation })}\right)=\beta_{0}+\beta_{1} \times \text { Period }
$$

where the Period is considered an explanatory variable in the logistic regression model and the two $\beta$ values the logistic regression coefficients of the model. Pr (Higher correlation) and $\operatorname{Pr}$ (Lower correlation) denote the "Higher" and "Lower" correlation probabilities, respectively. In this study, two distinct precincts, the central and west precincts in Tainan city, merged into one single unified administrative unit in 2004. These unpaired data were omitted and the total data from 348 townships were tested using logistic regression.

Modeling of the logistic regression was performed using SPSS 12. Global Moran's I statistic and local Moran's I statistic was calculated using Geoda (http://www. geoda.uiuc.edu/), an open source spatial analysis system, and visualized on LISA cluster maps using ArcMap 9.3.

\section{RESULTS}

Figure 2 displays the spatial clusters (hot spots and clod spots) as obtained using LISA statistic for the top 13 leading malignant neoplasms for both males and females in Taiwan during two time periods (1995-1998 and 2005-2008).

Table 1 summarizes the results from global autocorrelation statistics for the top 13 leading malignant neoplasms according to gender and in the two time periods (1995-1998 and 2005-2008) in Taiwan. The results of the global Moran's $I$ tests for most cases related to the leading malignant neoplasms are statistically significant, having a pseudo $p$-value smaller than 0.05 , and indicated spatial heterogeneity. However, opposing results (a pseudo $p$-value larger than 0.05) emerged in nine cases of which are pancreas cancer for males (1995-1998), nonHodgkin's lymphoma for males (1995-1998) and females (1995-1998 and 2005-2008), gallbladder and extrahepatic bile ducts cancer for males (1995-1998) and females (1995-1998 and 2005-2008), and leukemia for males (2005-2008) and females (1995-1998), respectively.

Table 2 summarizes the typology patterns, as calcu- 

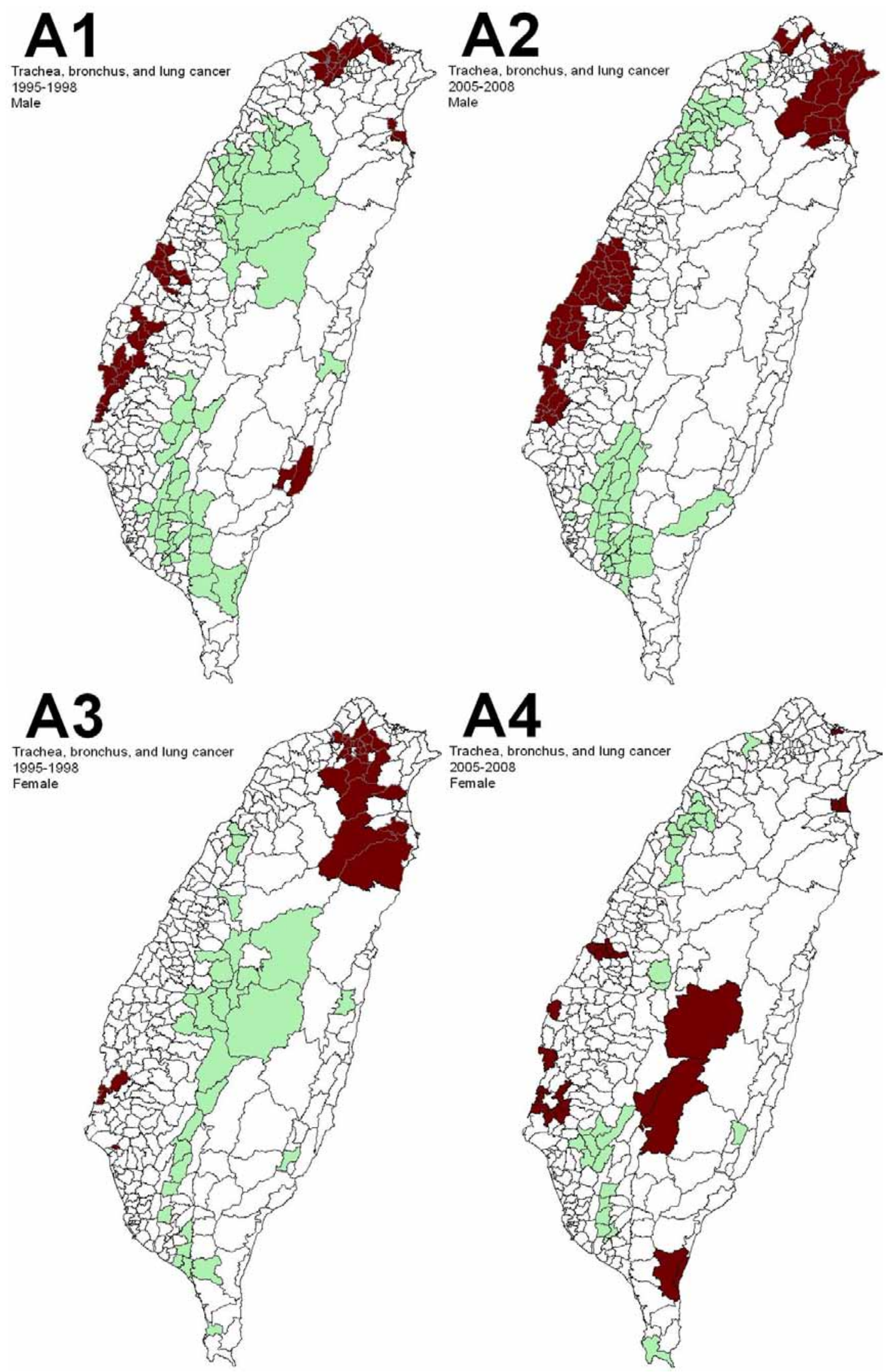
B1 1995-1998 Male

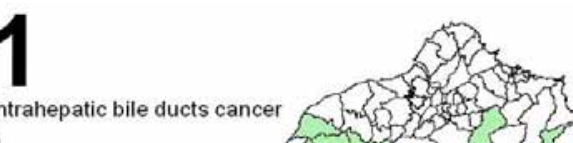

\section{B2}

Liver and intrahepatic bile ducts cancer 2005-2008 Male

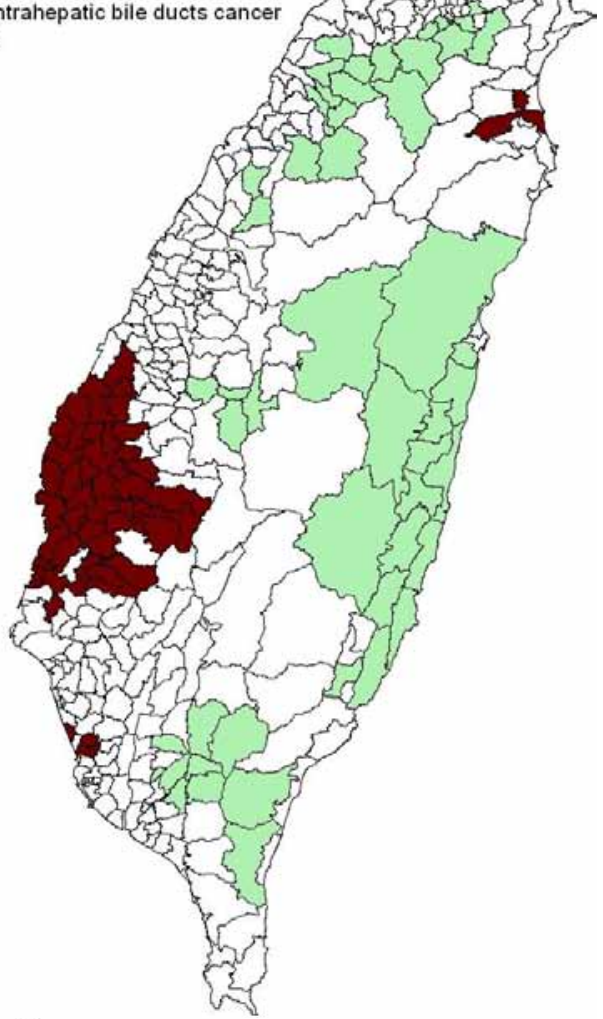

B3 1995-1998 Female

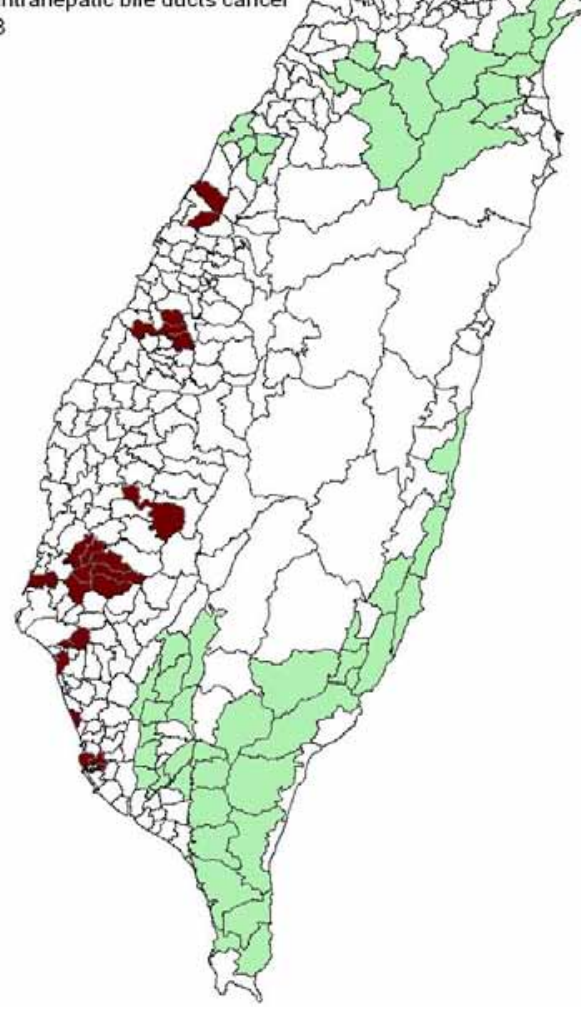

B4

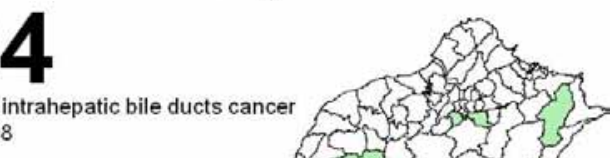
2005-2008

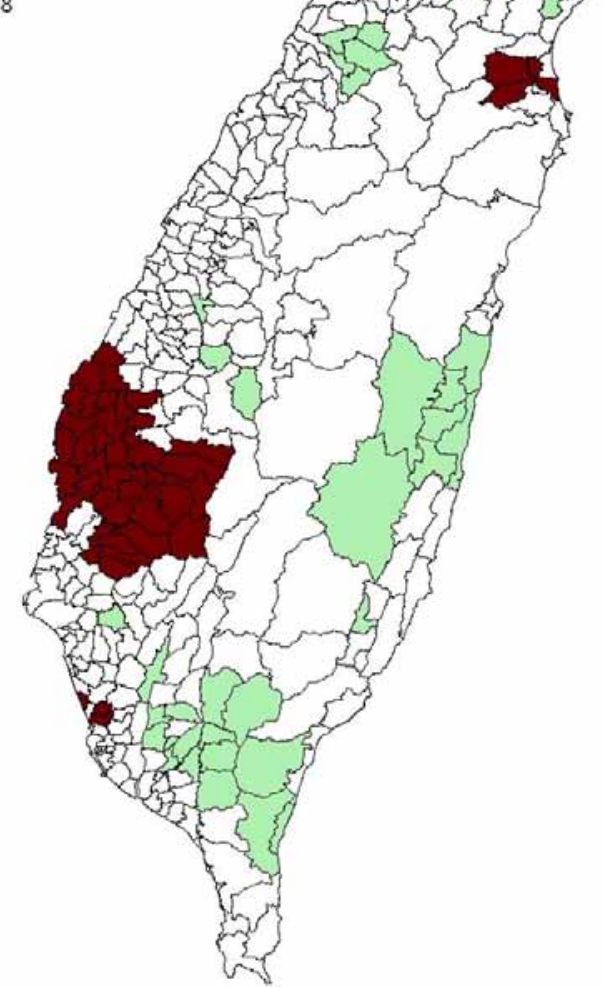



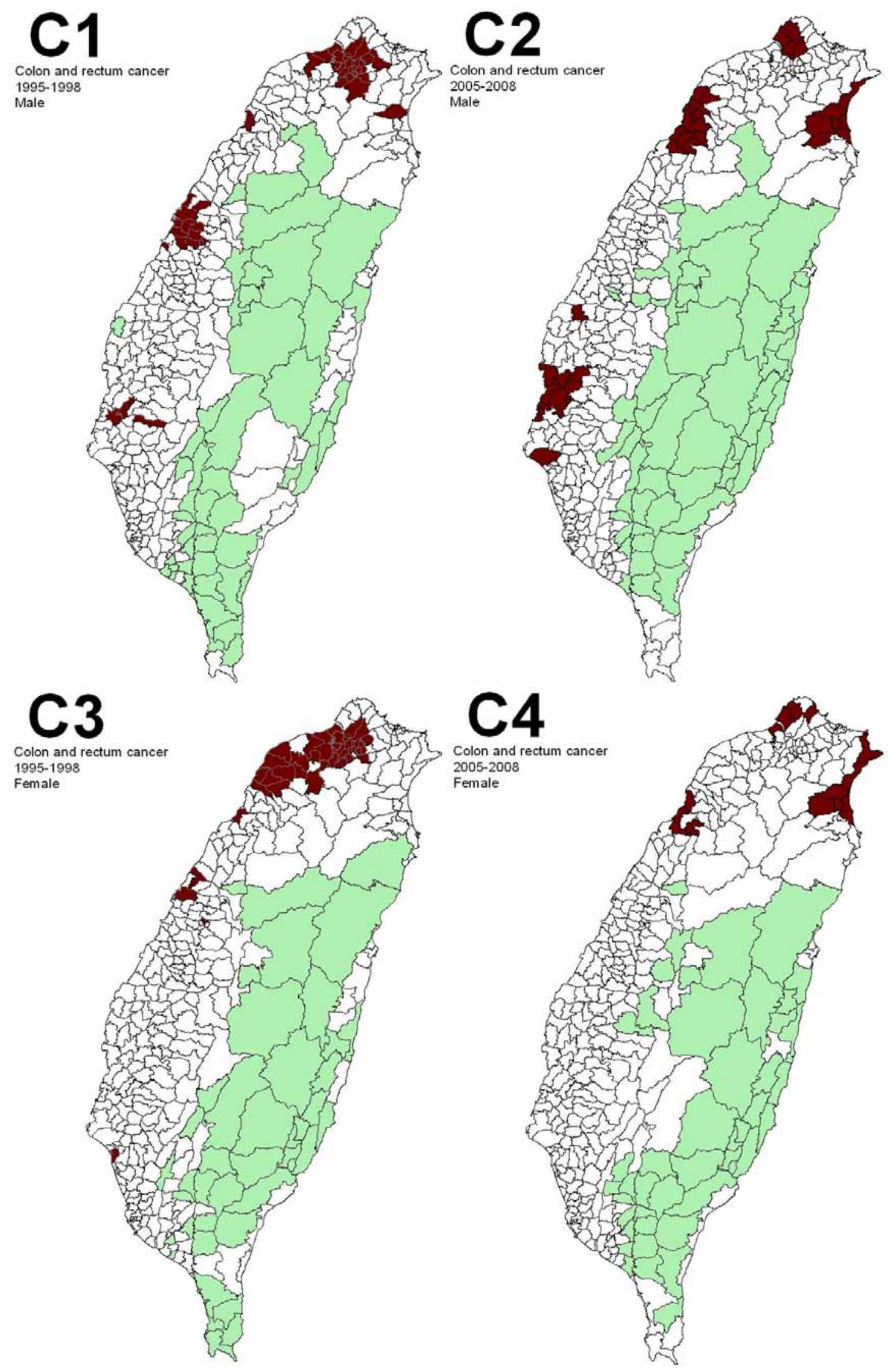

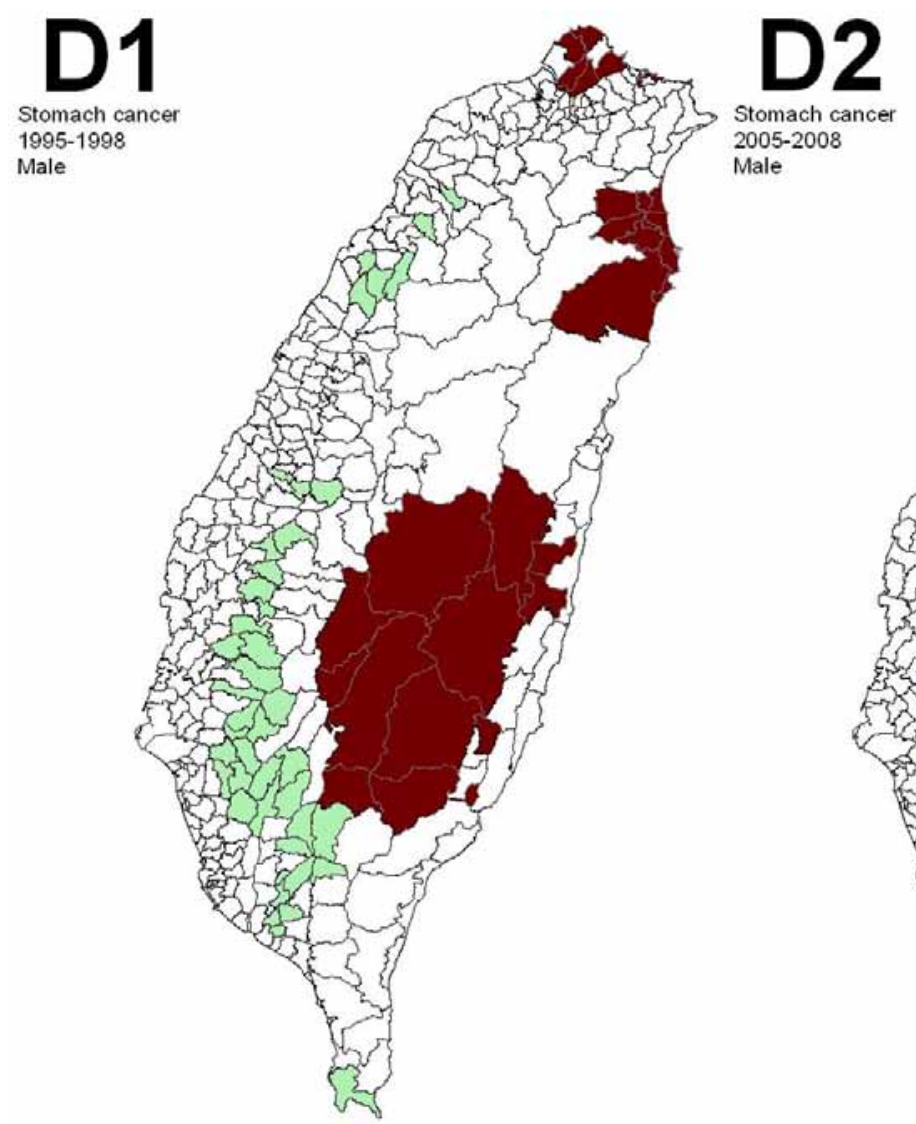

Male
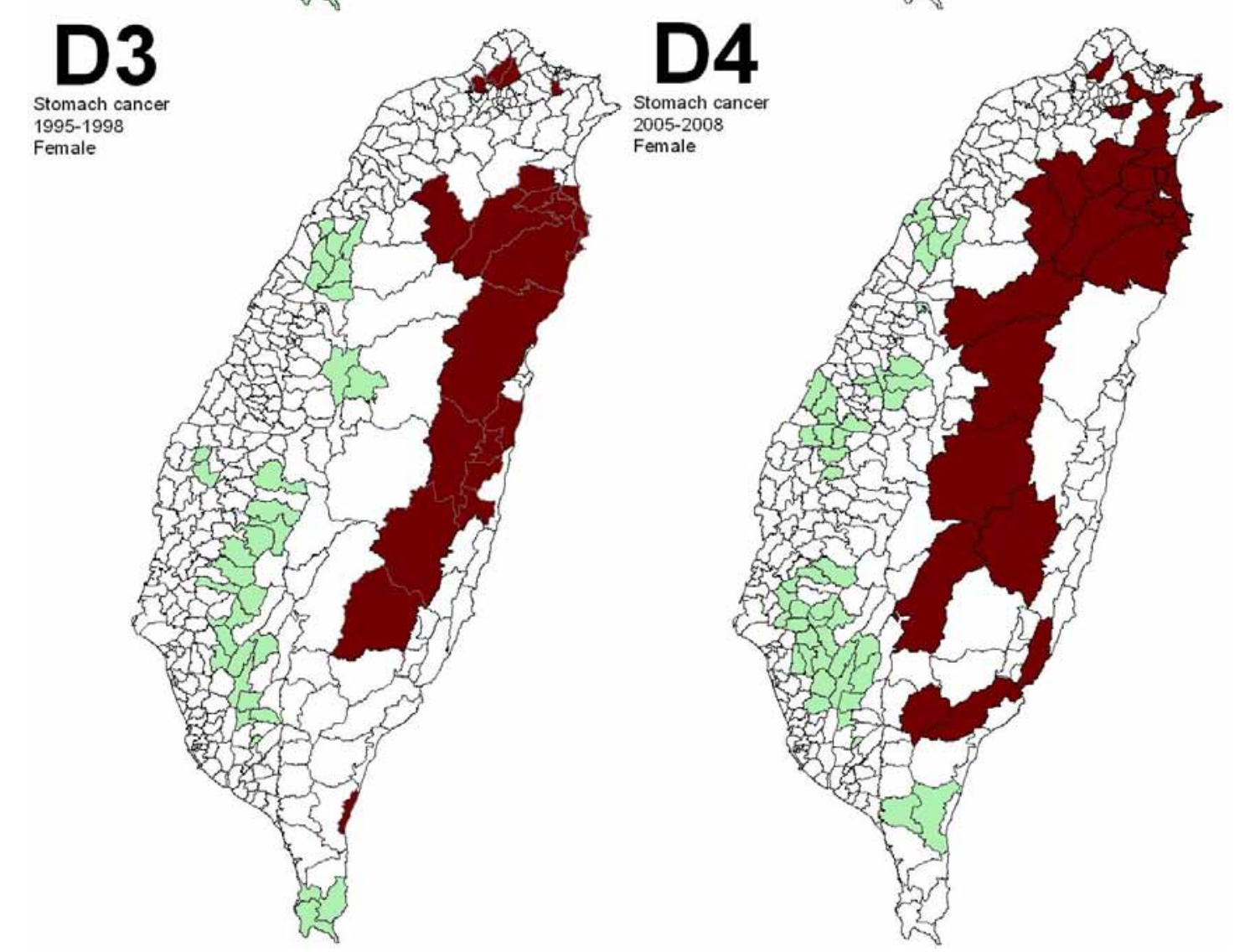

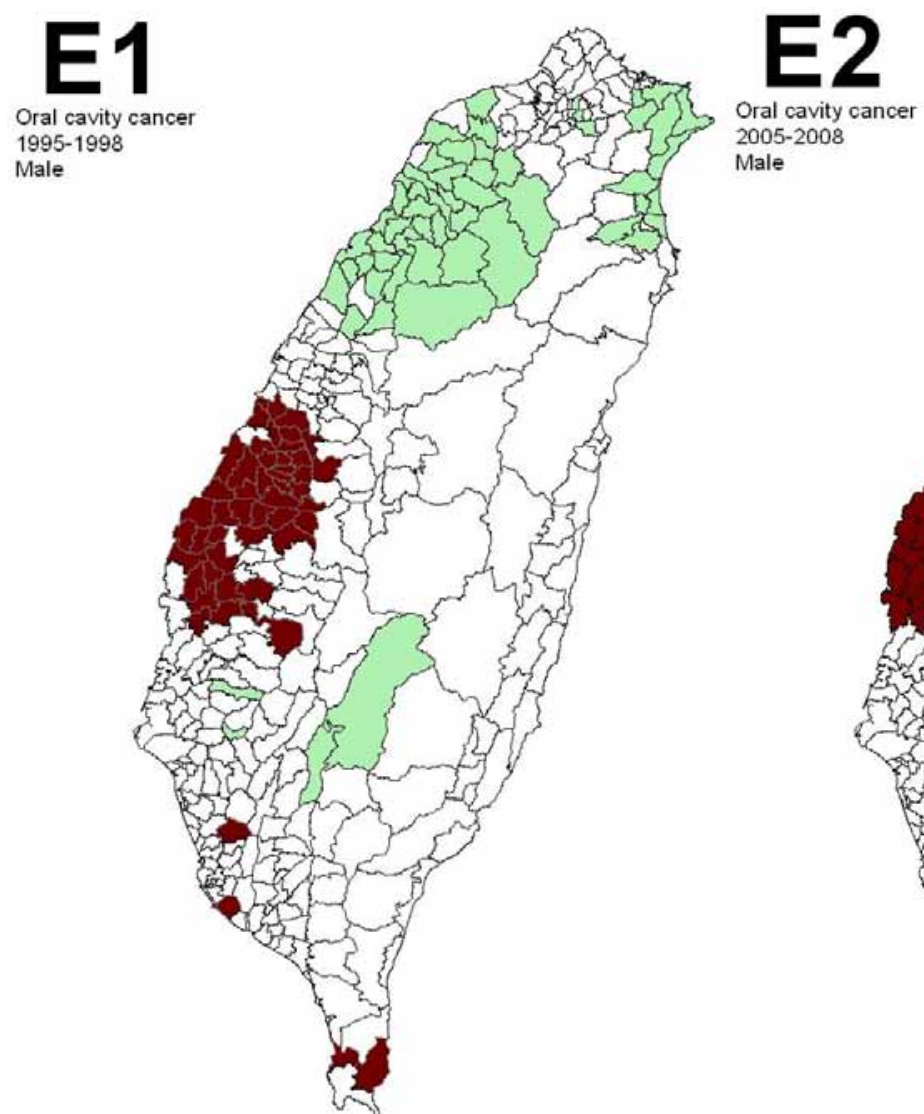

Male
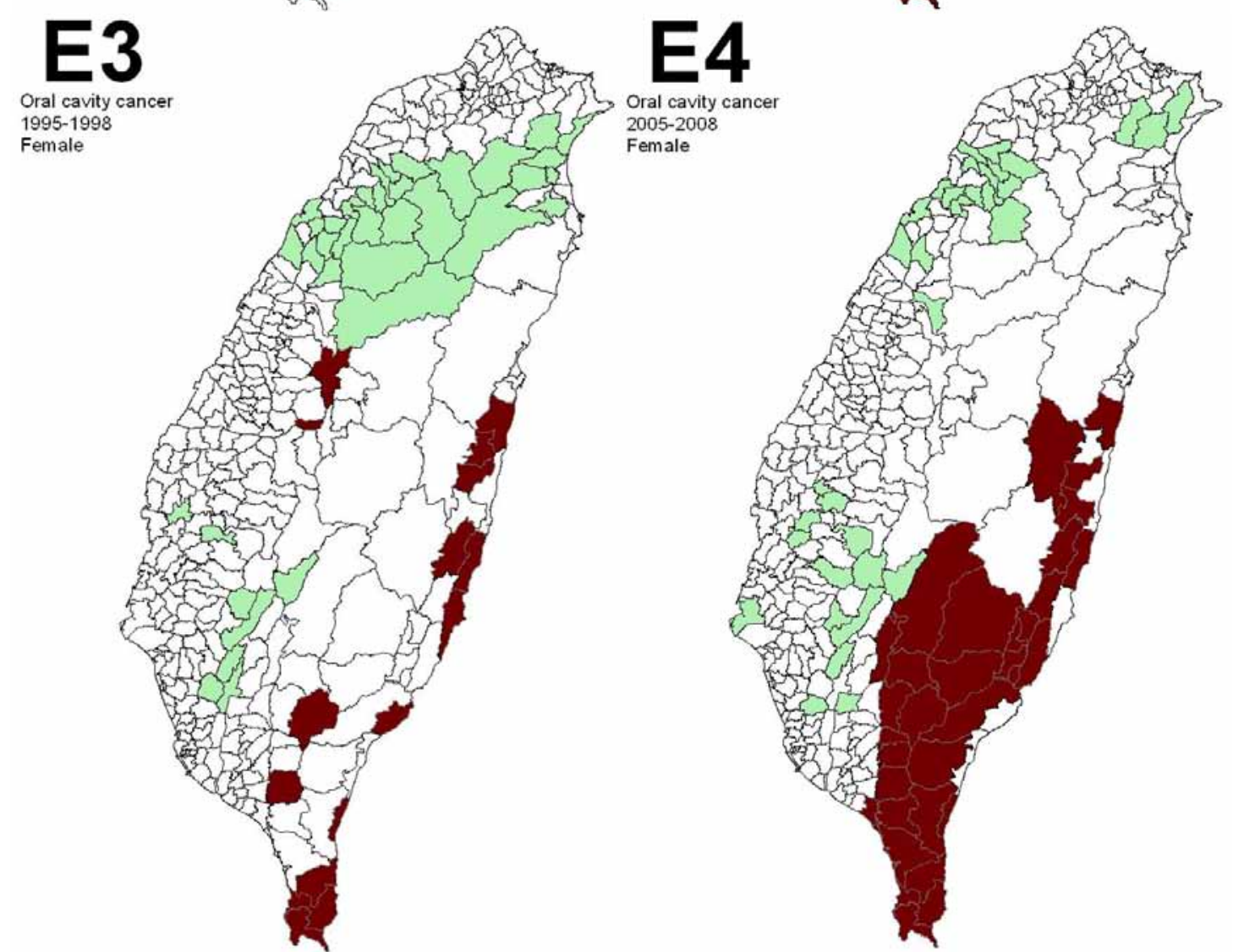

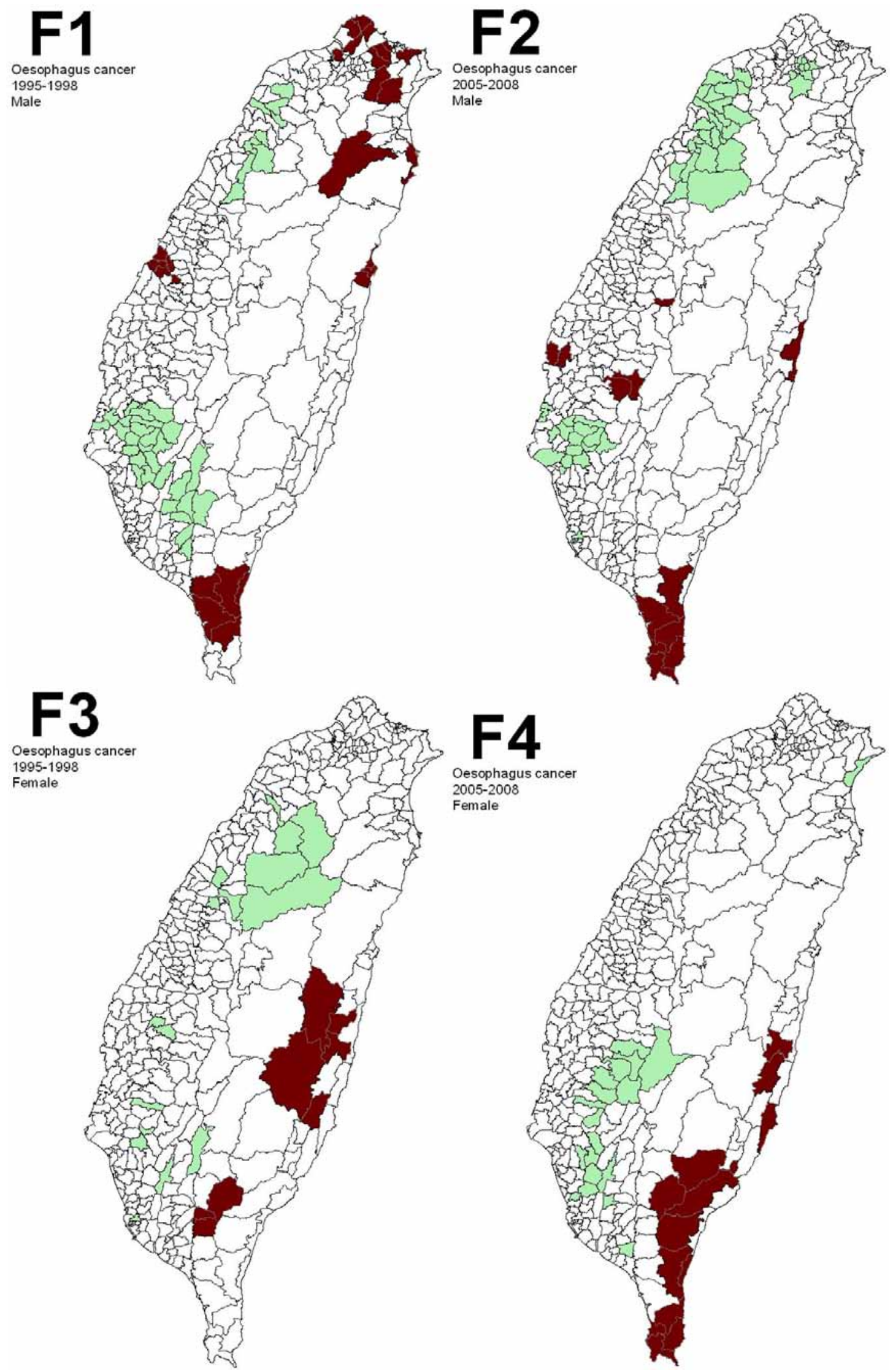

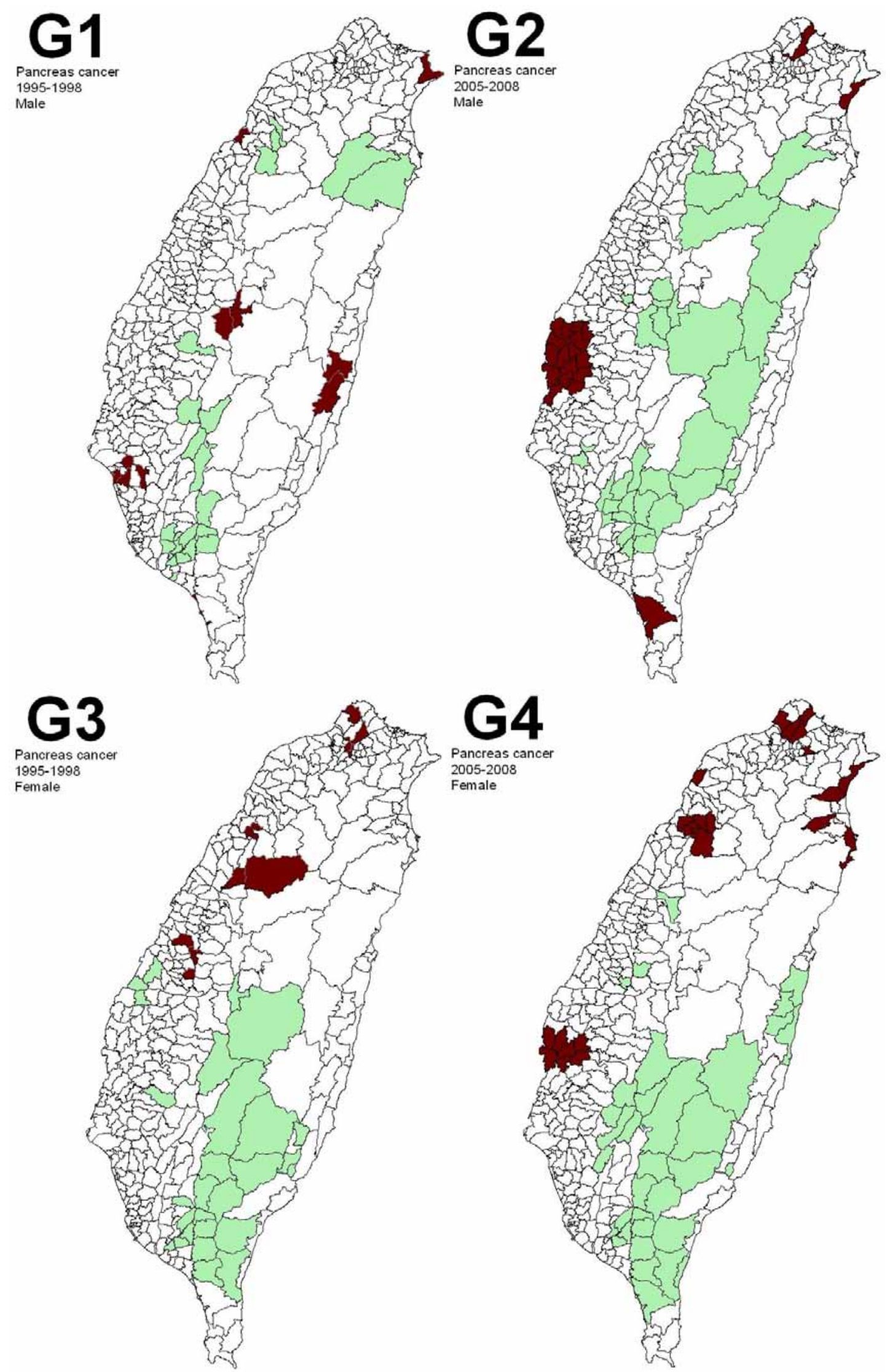

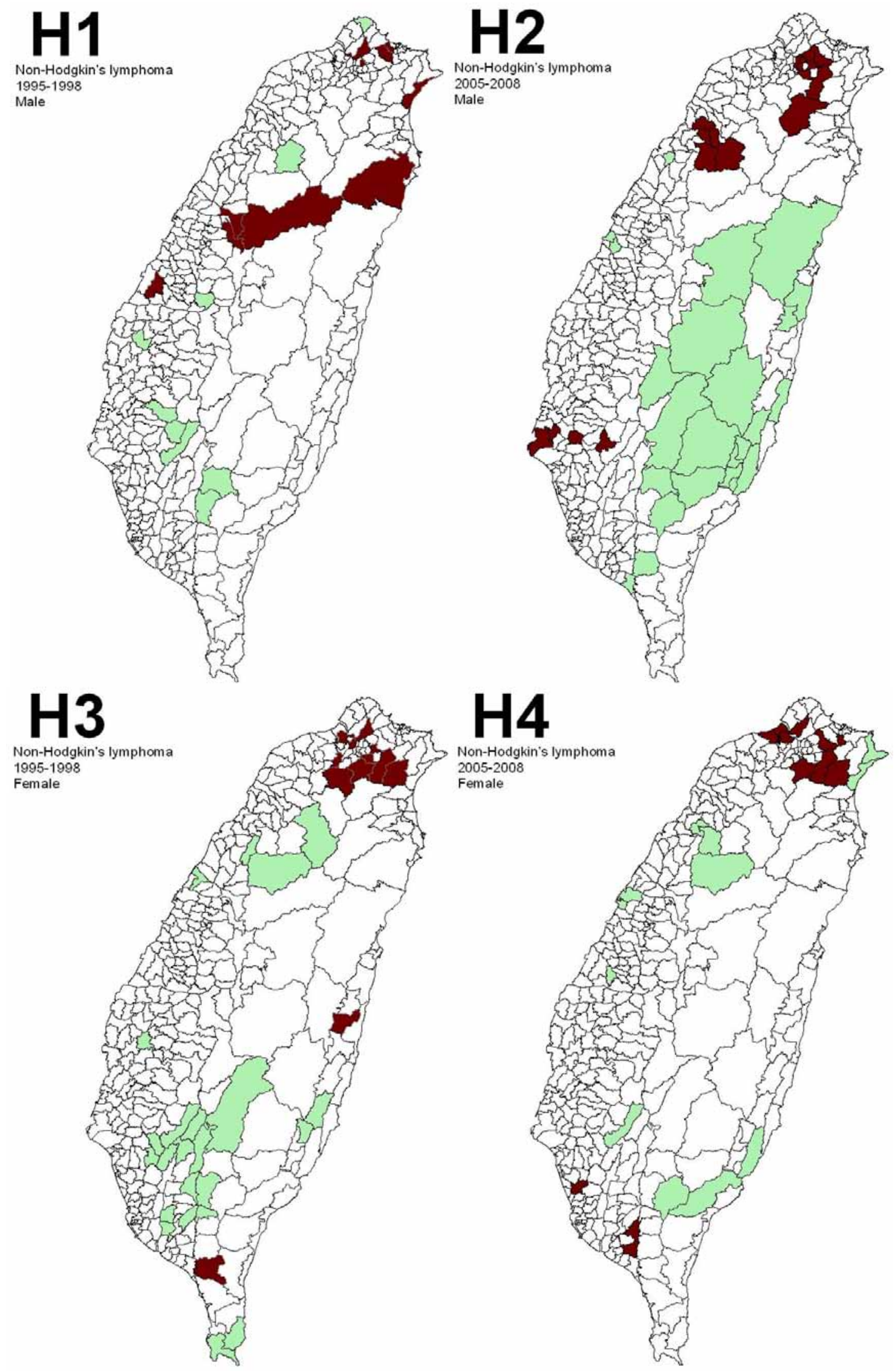


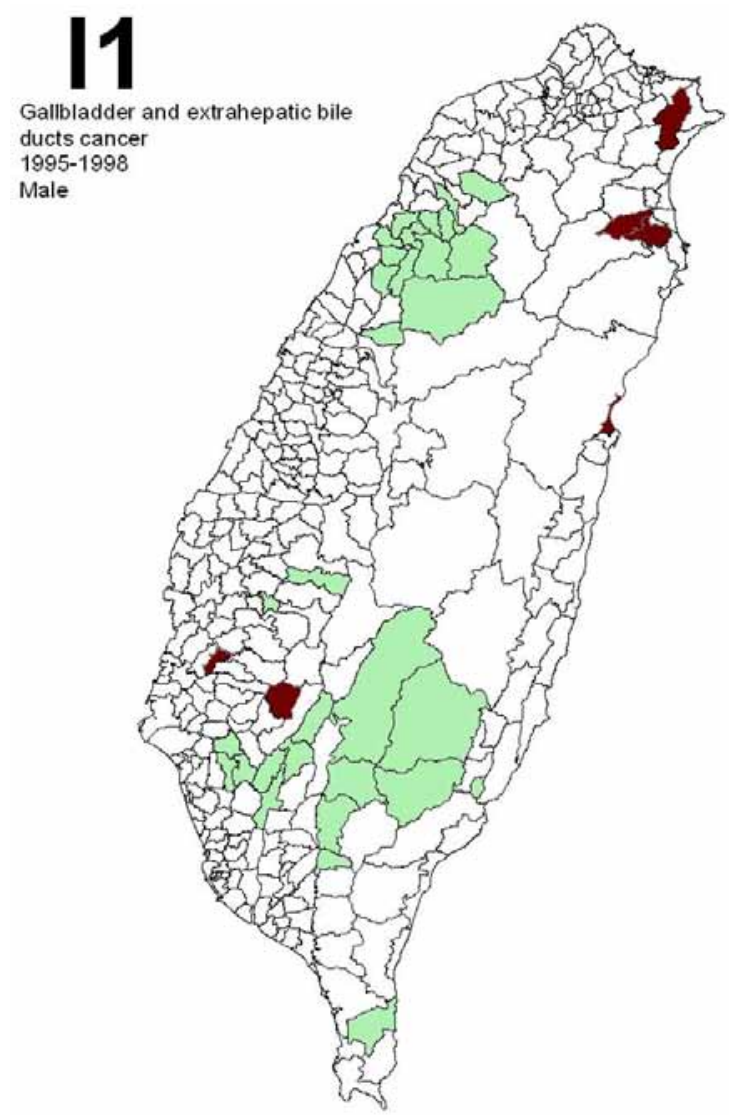
Gallbladder and extrahepatic bile
ducts cancer
2005-2008
Male
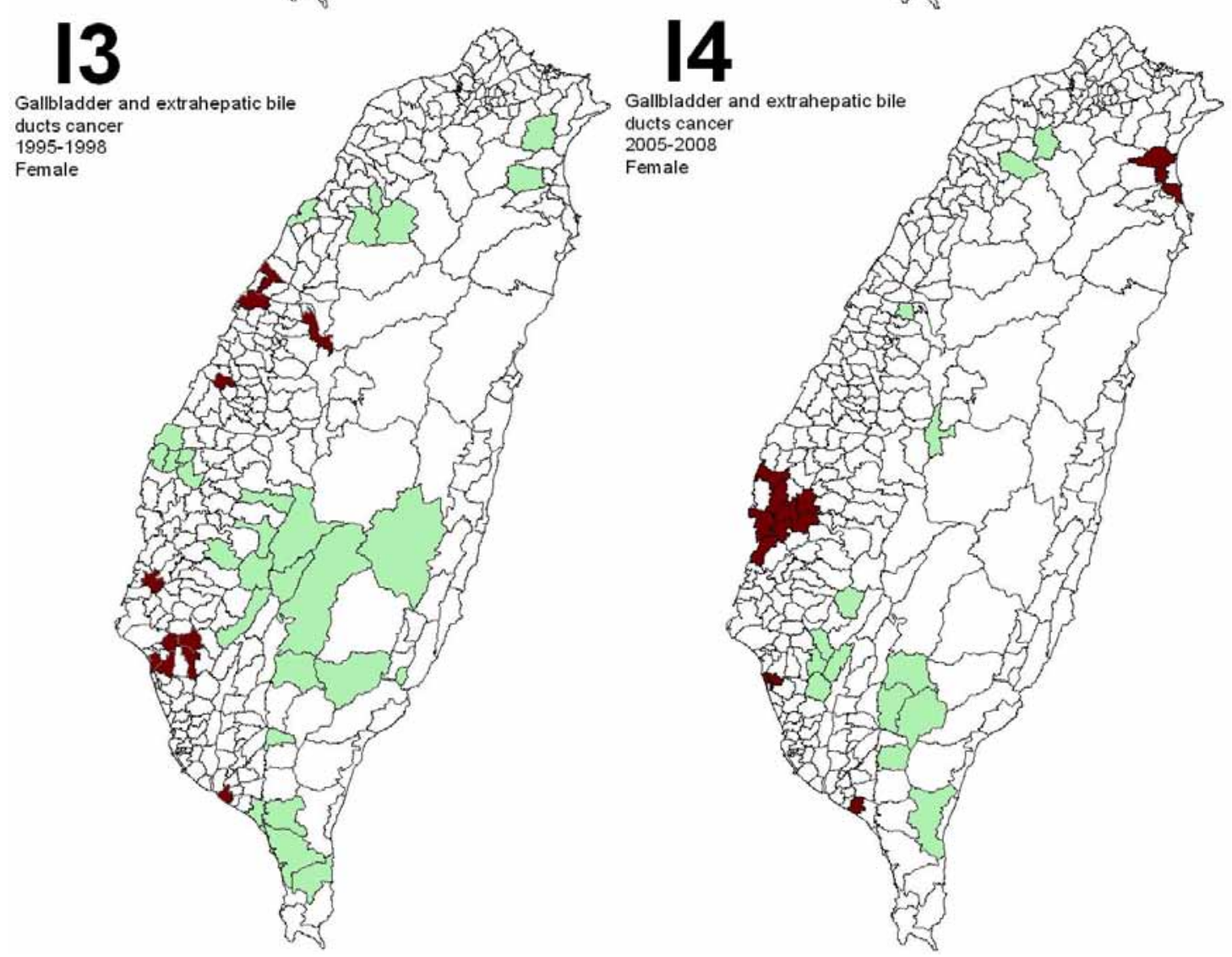

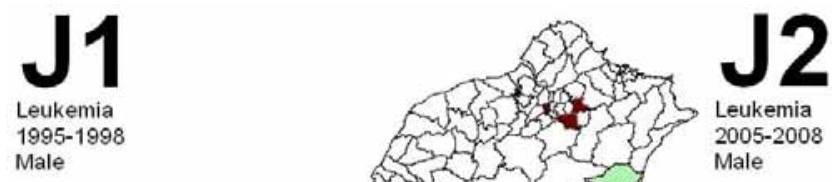

Male

Male
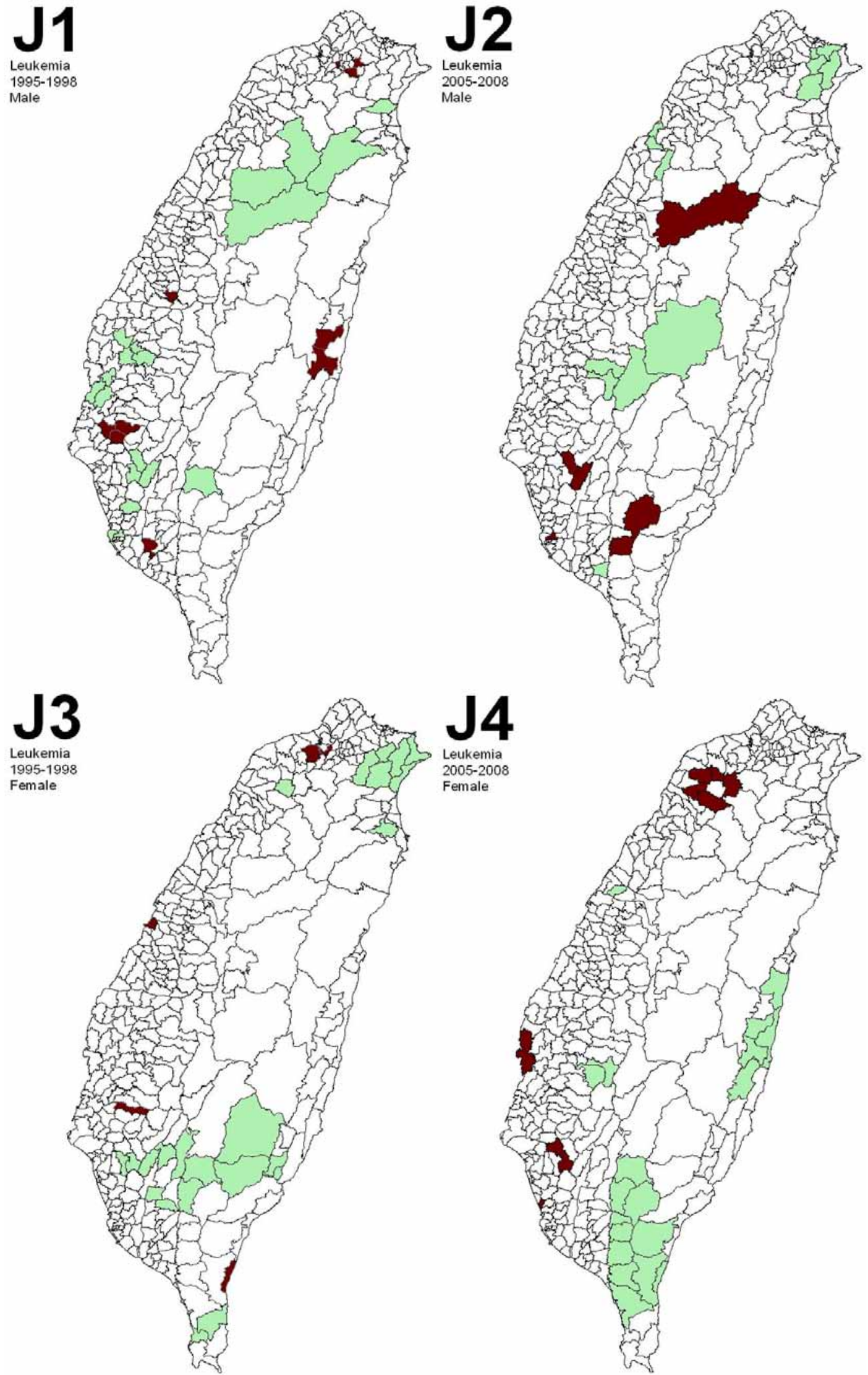

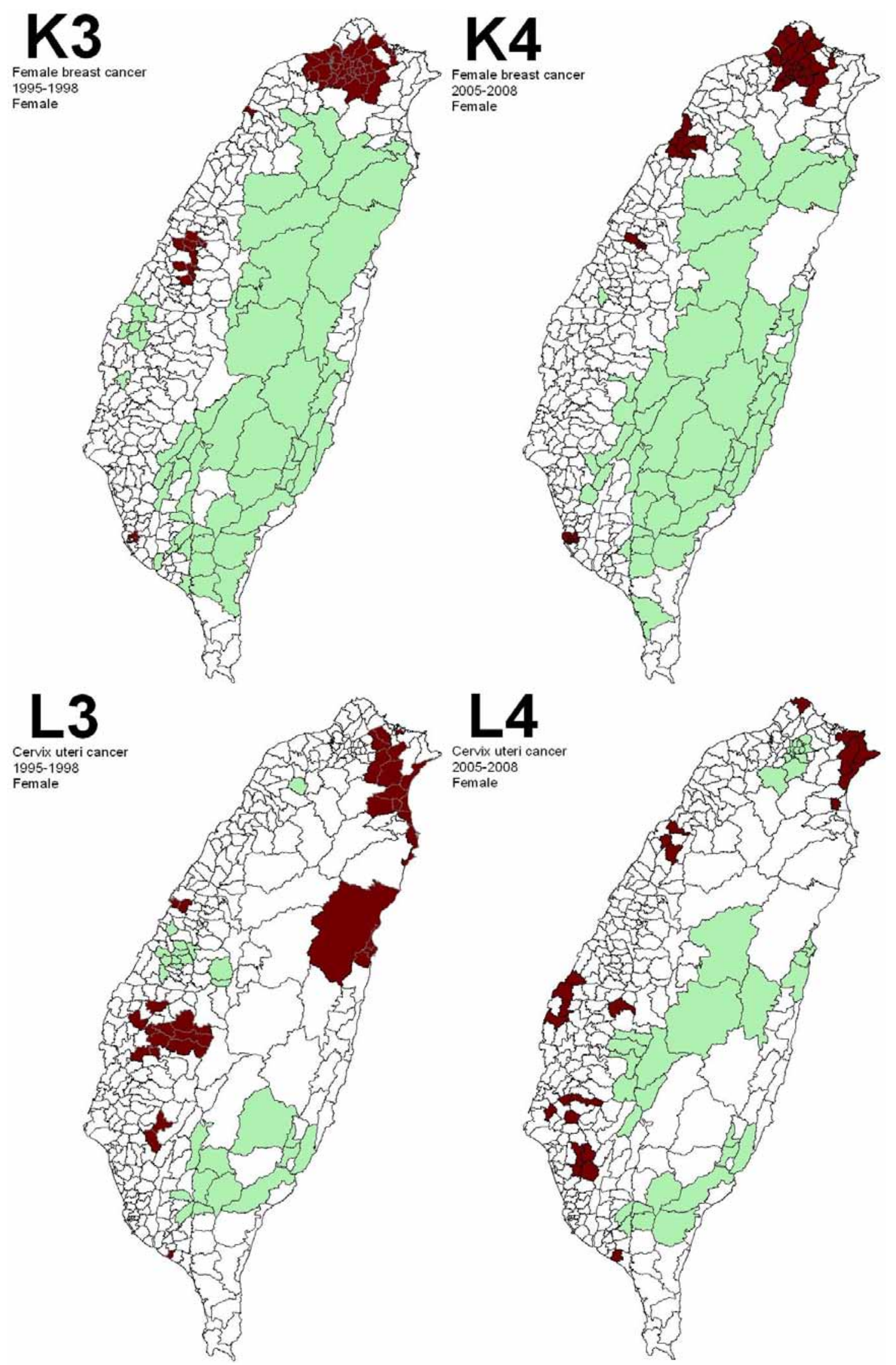


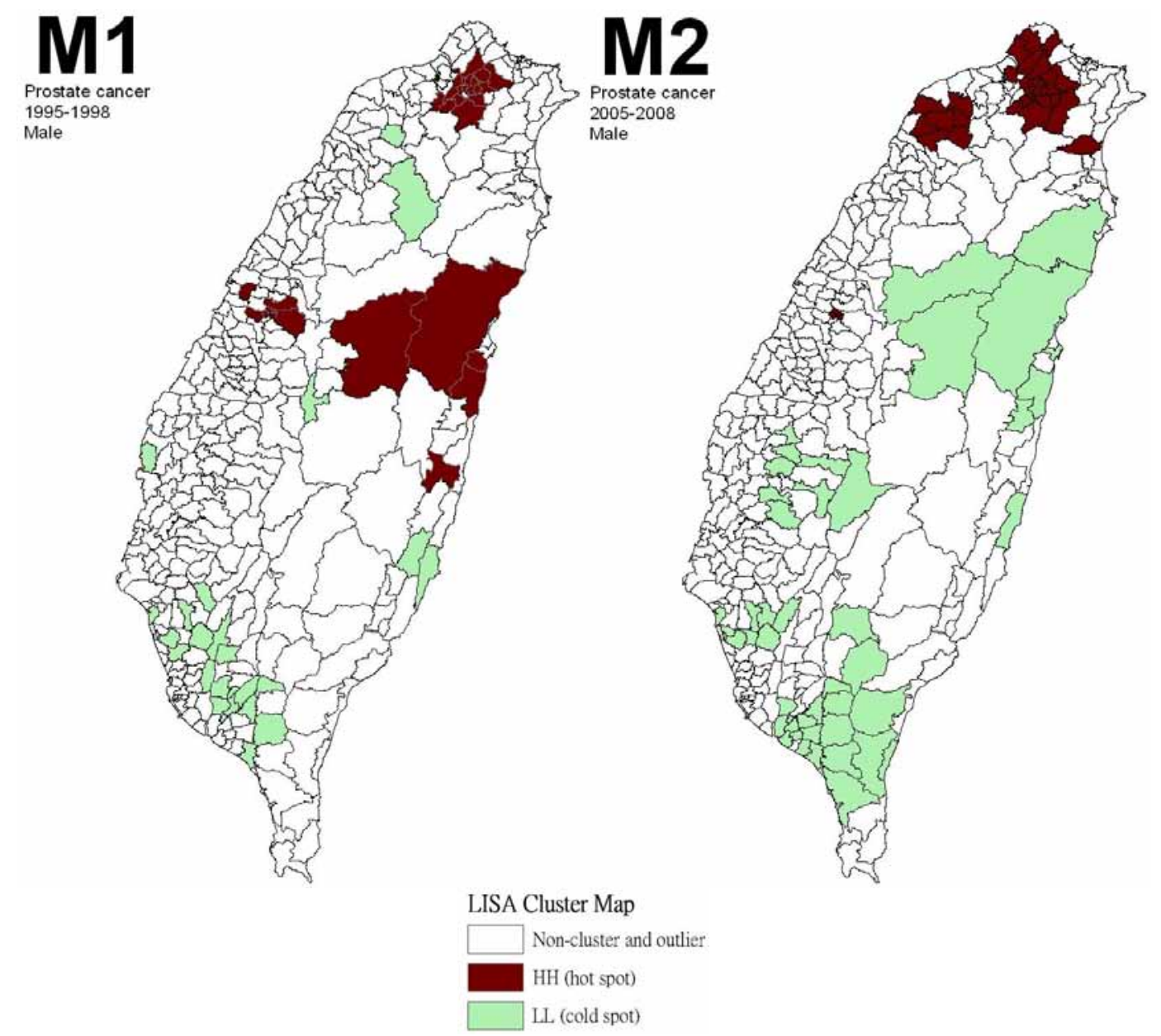

Figure 2. Spatial clusters of the 13 leading malignant neoplasms in Taiwan. Maps showing the spatial clusters of the 13 leading malignant neoplasms in Taiwan: A indicates trachea, bronchus, and lung cancer; B, liver and intrahepatic bile ducts cancer; C, colon and rectum cancer; D, stomach cancer; E, oral cavity cancer; F, oesophagus cancer; G, pancreas cancer; H, non-Hodgkin's lymphoma; I, gallbladder and extrahepatic bile ducts cancer; J, leukaemia; K, female breast cancer; L, cervix uteri cancer; M, prostate cancer. 1 indicates males within the period from 1995 to 1998 years; 2, males within the period from 2005 to 2008 years; 3, females within the period from 1995 to 1998; 4, females within the period from 2005 to 2008.

lated using LISA statistic, categorized as clusters or nonclusters at a z-score larger than +1.96 . It also compares the top 13 leading malignant neoplasms during the two time periods (1995-1998 and 2005-2008).

Dissimilarities between the spatial distribution patterns during the two periods (1995-1998 and 2005-2008) are not statistically significant ( $p$-value $>0.05$ ) in males for six out of eleven spatial clusters, and in females for ten of twelve spatial clusters. In males, there are dissimilarities for stomach cancer, oral cavity cancer, pancreas cancer, non-Hodgkin's lymphoma, and prostate cancer. In females, colon and rectum cancer, and pancreas cancer are dissimilar. Table 2 presents these findings.

\section{DISCUSSION}

Locations in close proximity tend to share similar attributes. According to Tobler (1979), "everything is related to everything else, and nearby things are more closely related to nearby things than to distant things" [33]. In epidemiology, a cluster becomes apparent when a number of health events occur which are situated close together in space and/or time. The evaluation of spatial distributions as a measure of disease risk may provide etiological insights [34]. Spatial autocorrelation is the relation between the values of a single variable attributable to the geographic arrangement of areal units on a map and can be used to determine the degree of spatial clustering [35,36]. In this study, local Moran's I statistic was used to measure the degree of spatial clustering and map the geographic patterns of the areal units. Spatial clustering of the leading cause of death (also called hot spots and cold spots) was identified by a z-score value larger than +1.96. In epidemiology, "hot spots" are 
Table 1. Global autocorrelation analysis of data for the 13 leading malignant neoplasms in Taiwan, according to gender, during 19951998 and 2005-2008.

\begin{tabular}{ccccc}
\hline & \multicolumn{3}{c}{ Moran's I } \\
\hline Leading malignant neoplasms (ICD code) & \multicolumn{2}{c}{ Male } & \multicolumn{2}{c}{ Female } \\
& $1995-1998$ & $2005-2008$ & $1995-1998$ & $2005-2008$ \\
\hline Trachea, bronchus, and lung cancer (ICD 162) & $0.38^{*}$ & $0.46^{*}$ & $0.17^{*}$ & $0.17^{*}$ \\
Liver and intrahepatic bile ducts cancer (ICD 155) & $0.45^{*}$ & $0.59^{*}$ & $0.34^{*}$ & $0.42^{*}$ \\
Colon and rectum cancer (ICD 153, 154) & $0.40^{*}$ & $0.52^{*}$ & $0.40^{*}$ & $0.49^{*}$ \\
Stomach cancer (ICD 151) & $0.34^{*}$ & $0.37^{*}$ & $0.22^{*}$ & $0.35^{*}$ \\
Oral cavity cancer (ICD 140, 141, 143-146, 148, 149) & $0.43^{*}$ & $0.68^{*}$ & $0.09^{*}$ & $0.68^{*}$ \\
Oesophagus cancer (ICD 150) & $0.24^{*}$ & $0.22^{*}$ & $0.07^{*}$ & $0.25^{*}$ \\
Pancreas cancer (ICD 157) & 0.05 & $0.18^{*}$ & $0.07^{*}$ & $0.22^{*}$ \\
Non-Hodgkin’s lymphoma (ICD 200, 202, 203) & 0.02 & $0.07^{*}$ & 0.05 & 0.05 \\
Lallbladder and extrahepatic bile ducts cancer (ICD 156) & 0.06 & $0.14^{*}$ & 0.05 & 0.04 \\
Feukaemia (ICD 204-208) & $0.08^{*}$ & 0.04 & 0.01 & $0.08^{*}$ \\
Cervix uteri cancer (ICD 179, 180) & n.d. & n.d. & $0.52^{*}$ & $0.53^{*}$ \\
Prostate cancer (ICD 185) & n.d. & n.d. & $0.24^{*}$ & $0.26^{*}$ \\
\hline
\end{tabular}

n.d.: no detection. ${ }^{*}$ : A pseudo $p$-value smaller than 0.05 .

Table 2. Logistic regression model comparisons of the 13 leading malignant neoplasms in Taiwan, during 1995-1998 and $2005-2008$.

\begin{tabular}{|c|c|c|c|c|}
\hline \multirow{2}{*}{ Leading malignant neoplasms (ICD code) } & \multicolumn{2}{|c|}{ Male } & \multicolumn{2}{|c|}{ Female } \\
\hline & $p$-value & description & $p$-value & description \\
\hline Trachea, bronchus, and lung cancer (ICD 162) & 0.245 & similarity $^{\mathrm{a}}$ & 0.21 & similarity $^{\mathrm{a}}$ \\
\hline Colon and rectum cancer (ICD 153, 154) & 0.492 & similarity $^{\mathrm{a}}$ & 0.019 & dissimilarity $^{\mathrm{a}}$ \\
\hline Stomach cancer (ICD 151) & 0.034 & dissimilarity $^{\mathrm{a}}$ & 0.053 & similarity $^{\mathrm{a}}$ \\
\hline Oesophagus cancer (ICD 150) & 0.844 & similarity $^{\mathrm{a}}$ & 0.266 & similarity $^{\mathrm{a}}$ \\
\hline Pancreas cancer (ICD 157) & 0.029 & dissimilarity & 0.047 & dissimilarity $^{\mathrm{a}}$ \\
\hline Non-Hodgkin’s lymphoma (ICD 200, 202, 203) & 0.006 & dissimilarity & 0.179 & similarity \\
\hline Gallbladder and extrahepatic bile ducts cancer (ICD 156) & 0.409 & similarity & 0.197 & similarity \\
\hline Leukaemia (ICD 204-208) & 0.137 & similarity & 0.781 & similarity \\
\hline Cervix uteri cancer (ICD 179, 180) & & n.d. & 0.84 & similarity $^{\mathrm{a}}$ \\
\hline Prostate cancer (ICD 185) & 0.007 & dissimilarity $^{\mathrm{a}}$ & & n.d. \\
\hline
\end{tabular}

n.d.: no detection. ${ }^{a}$ : A comparison of the two periods during which all of Moran's test results are clusters (results based on Table 1).

considered interesting because of their correlation to aetiology. This study, therefore, focuses on the spatial locations of 13 leading malignant neoplasms. Information about spatial location is useful for detecting risk from a spatial point of view. A more detailed survey of these identified "hot spots" may provide important clues on risk factors for these diseases.

The modifiable areal unit problem (MAUP) is a phenomenon whereby analysis of the same data provides different results, grouped into different sets of areal units. The MAUP can be subdivided into two separate effects that usually occur simultaneously during the analysis of aggregated data. The scale effect causes variation in statistical results according to different levels of aggregation. An association between variables, therefore, depends on the sizes of the areal units of the reported data. Generally, correlation increases as the size of the areal unit increases. The zone effect describes variations in correlation statistics caused by the regrouping of data into different configurations, but with the same scale. The MAUP occurs because spatial processes generating the observed data may exist within certain scales, and for particular areal units. These may be reflected more or less accurately by the boundaries in use [37]. Manley et 
al. (2006) concluded that MAUP is not really a problem, but rather, a resource. Data at different scale levels can enable the identification of processes operating within different scales. It is clear that it is not possible to define an ideal single census geography that captures all of the processes for all variables [37]. Furthermore, the internal composition of given areal units may not be homogeneous, particularly for disease distribution. Matisziw et al. (2008) have suggested that down-scaling the spatial structure of polygonal units could provide valuable information pertaining to the spatial distribution of disease [38]. In this study, administrative government regions are almost similar but not completely consistent in the two periods (1995-1998 and 2005-2008). This was to some degree due to the merging of the central and west districts in Tainan city merging into one unit in 2004. The use of only one scale to estimate spatial distribution patterns, although still a cluster comparison, would be more convenient; however, bias could be caused by using a non-realistic spatial boundary. An ideal process would be to calculate the spatial autocorrelation coefficients (such as the z-scores) based on realistic boundaries (two scales for shape files that represented 350 townships in 1995-1998 and 349 townships in 2005-2008, respectively) and then omit the values of autocorrelation coefficients that were non-paired data from the comparison of the two periods within the administrative regions.

The local spatial autocorrelation coefficients can be tested for statistical significance under two rather different model assumptions. The first is the classical statistical assumption of normality, whereby it is assumed that the observed value of the coefficient is the result of the set of z-score values being independent and identically distributed drawings from a normal distribution, implying that variances are constant across the region. The second model is one of randomization, whereby the observed pattern of the set of z-score values is assumed to be just one realization from all possible random permutations of the observed values across all the zones. Both models have important weaknesses. For example, there is an underlying population size variation and a lack of homogeneity of probabilities; however these models are widely implemented in software packages to provide estimates of the significance of observed results. In the case of the randomization model, many software packages generate a set of $N$ random permutations of the input data, where $N$ is user specified. For each simulation run, index values are computed and the set of such values are used to provide a pseudo-probability distribution for the given problem, against which the observed value can be compared. A z-transform of the coefficients under normality or randomization assumptions is distributed approximately as $\mathrm{N}(0,1)$; hence, this may be com- pared to percentage points of the normal distribution to identify particularly high or low values [39]. In this study, the comparison of databases from the two periods (1995-1998 and 2005-2008) was addressed by the Taiwan Cancer Registry and the Taiwan National Health Insurance agencies, respectively. Although the two databases have a referenced value with high validity and reliability, this case was defined with the same diagnostic criteria (ICD 9 CM) and a world standard population in 1976 to calculate the morbidity rate. However, the estimated morbidity rates derived from the two databases cannot be directly compared with one another. Our suggested resolution is to change the morbidity rate into a z-transform by using a spatial autocorrelation calculation with a randomization of 999 permutations, and this then makes two z-transform comparisons feasible. Binominal variable logistic regression models were used to distinguish spatial distribution patterns that addressed the two periods (1995-1998 and 2005-2008).

Z-scores for the LISA method were calculated using the logistic regression model and results for various leading malignant neoplasms during two periods (19951998 and 2005-2008) were compared. However, the constraint condition for spatial clustering comparison (such as global Moran's tested clusters on both sides) are required to be satisfied before calculating the logistic regression for purposes of comparison. Based on this constraint, the results demonstrate statistically significant differences for stomach cancer (in males), oral cavity cancer (in males), prostate cancer (in males), colon and rectum cancer (in females), and pancreas cancer (in females). Another eleven compared cases were not significantly different. The null hypothesis is, therefore, accepted. The accepted null hypothesis results indicate that the common spatial factor(s) may interact with both periods.

Few previous ecological studies relate to malignant neoplasms and their correlation to risk factors in Taiwan, although oral cancer and stomach cancer have been documented and are discussed briefly below. It is hoped that this assessment of the spatial clustering of Taiwan's leading malignant neoplasms can contribute to the study of spatial epidemiology.

Two separate groups identified clusters of areas showing elevated mortality from oral cavity cancer in females in the aboriginal townships in eastern Taiwan. The habits of cigarette smoking, alcohol drinking and betel nut chewing had higher prevalence in aboriginal women in eastern Taiwan than in women in other regions $[40,41]$. Chiang et al. suggested that high-risk areas of oral cancer incidence in males closely coincided with spatial distribution of heavy-metal pollution in soils (such as chromium and nickel) in central Taiwan [42]. In 
this study, oral cavity cancer clusters for each gender were calculated using the LISA statistic. Results identify clear spatial clustering in central Taiwan, for males, and eastern Taiwan for females, among Taiwanese aboriginal townships. These observations, therefore, support the results described in previous studies. However, according to our results, the two periods (1995-1988 and 20052008), show dissimilarity in the spatial distribution of oral cavity cancer in males. Spatial risks affecting oral cancer morbidity in males reveal space-time changes. These findings could be interpreted as the changing disease clusters over time, are due to the changes of exposure conditions to metal pollutant and leading to the results of a variation of virulence. Further investigation is therefore warranted.

Several meta-analyses identified a strong and consistent association between $H$. pylori infection and noncardiac gastric cancer [43-46]. The ecological study in Taiwan suggests an association between this infection and gastric cancer. H. pylori infection in early childhood may be a key issue and, it appears, a long induction time is required for gastric carcinogenesis. High gastric cancer mortality areas are clustered in the aboriginal townships where the prevalence of $H$. pylori is high [40, 47]. Our results are similar to these previous studies. Stomach cancer clusters for males and females are located in the Taiwanese aboriginal townships, and a new carcinogen cluster was identified in the northern coastal region of Taiwan. This is worthy of further investigation. However, the two periods (1995-1988 and 2005-2008) show dissimilarity in the spatial distribution of gastric cancer in males. Spatial risks affecting gastric cancer morbidity in males reveal space-time changes. By changing disease clusters over time, a possible reason is due to the changes of prevalence ranges of $H$. pylori or increased in the interference of other risks in the study area. Further investigation is therefore warranted.

\section{CONCLUSIONS}

A method which combines LISA statistics and logistic regression is an effective tool for the detection of spacetime patterns with discontinuous data. Similarity is a result of unchangeable condition in disease risks. Conversely, dissimilarity is deemed a significant change of morbidity risks over the studied periods. This enables planners to assess spatial risk factors and to determine the most advantageous types of health care policies for the planning and implementation of health care services. These issues can greatly improve the performance and effectiveness of health care services and also provide a clear outline for better understanding of the results in depth.

\section{ACKNOWLEDGEMENTS}

The authors would like to thank Taiwan's Department of Health for providing the National Health Insurance and Bureau of Health Promotion databases.

\section{REFERENCES}

[1] Gesler, W. (1986) The uses of spatial analysis in medical geography: A review. Social Science \& Medicine, 23, 963-973. doi:10.1016/0277-9536(86)90253-4

[2] Cuzick, J. and Edwards, R. (1990) Spatial clustering for inhomogeneous populations. Journal of the Royal Statistical Society, 52, 73-104.

[3] Cressie, N.A.C. (1993) Statistics for spatial data. Wiley, New York.

[4] Legendre, P. and Legendre, L. (1998) Numerical ecology. 2nd English Edition, Elsevier, Amsterdam.

[5] Fortin, M.J. (1999) Spatial statistics in landscape ecology. In: Klopatek, J.M. and Gardner, R.H., Eds., Landscape Ecological Analysis: Issues and Applications, SpringerVerlag, New York, 253-279. doi:10.1007/978-1-4612-0529-6_12

[6] Tsai, P.J., Lin, M.L., Chu, C.M. and Perng, C.H. (2009) Spatial autocorrelation analysis of health care hotspots in Taiwan in 2006. BMC Public Health, 9, 464. doi:10.1186/1471-2458-9-464

[7] Anselin, L. (1995) The local indicators of spatial association-LISA. Geographical Analysis, 27, 93-115. doi:10.1111/j.1538-4632.1995.tb00338.x

[8] Getis, A. and Ord, J.K. (1992) The analysis of spatial association by use of distance statistics. Geographical Analysis, 24, 189-206. doi:10.1111/j.1538-4632.1992.tb00261.x

[9] Getis, A. and Ord, J.K. (1996) Local spatial statistics: An overview. In: Longley, P. and Batty, M., Eds., Spatial Analysis: Modeling in A GIS Environment, John Wiley \& Sons, New York, 261-277.

[10] Knox, E.G. (1964) The detection of space-time interaction. Appied Statistics, 13, 25-29. doi: $10.2307 / 2985220$

[11] Mantel, N. (1967) The detection of cancer clustering and the generalized regression approach. Cancer Research, 27, 209-220.

[12] Jacquez, G.M. (1996) A $k$ nearest neighbor test for space-time interaction. Statistics in Medicine, 15, 19351949.

doi:10.1002/(SICI)1097-0258(19960930)15:18<1935::AI D-SIM406>3.0.CO;2-I

[13] Kulldorff, M. and Nagarwalla, N. (1995) Spatial disease clusters: Detection and inference. Statistics in Medicine, 14, 799-810. doi:10.1002/sim.4780140809

[14] Kulldorff, M. (1997) A spatial scan statistic. Communication in Statistic: Theory and Methods, 26, 1481-1496. doi:10.1080/03610929708831995

[15] Kulldorff, M. (1999) Spatial scan statistics: Models, calculations, and applications. In: Glaz, J. and Balakrishnan, N., Eds., Scan Statistics and Applications, Birkhäuser, Boston, 303-322. doi:10.1007/978-1-4612-1578-3_14

[16] Neill, D.B., Moore, A.W. and Cooper, G.F. (2006) A Bayesian spatial scan statistic. Advances in Neural In- 
formation Processing Systems, 18, 1003-1010.

[17] Greenlee, R.T., Murray, T., Bolden, S. and Wingo, P.A. (2000) Cancer statistics. A Cancer Journal for Clinicians, 50, 7-33. doi:10.3322/canjclin.50.1.7

[18] Adami, H.O., Hunter, D. and Trichopoulos, D. (2002) Textbook of cancer epidemiology. Oxford University Press, New York.

[19] Parkin, D.M., Whelan, S.L., Ferlay, J., Teppo, L. and Thomas, D.B. (2002) Cancer incidence in five continents. IARC Scientific Publications, Lyon.

[20] Frank, S.A. (2007) Dynamics of cancer: Incidence, inheritance, and evolution. Princeton University Press, Princeton.

[21] National Health Insurance (2007) Statistical annual report of medical care 2005. National Health Insurance (Taiwan), Taipei.

[22] National Health Insurance (2008) Statistical annual report of medical care 2006. National Health Insurance (Taiwan), Taipei.

[23] National Health Insurance (2009) Statistical annual report of medical care 2007. National Health Insurance (Taiwan), Taipei.

[24] National Health Insurance (2010) Statistical annual report of medical care 2008. National Health Insurance (Taiwan), Taipei.

[25] Ministry of the Interior (2009) The demographic database. http://www.moi.gov.tw/stat/index.aspx

[26] Ahmad, O.E., Boschi-Pinto, C., Lopez, A.D., Murray, C.J.L., Lozano, R. and Inoue, M. (2000) Age standardization of rates: A new WHO standard (GPE discussion paper series, No. 31). World Health Organization Press, Geneva.

[27] Liaw, Y.P., Chen, C.J., Lee, W.C. and Hsu, S.Y. (2003) The construction and use of the electric atlas of cancer mortality and incidence in Taiwan. Taiwan Journal of Public Health, 22, 227-236.

[28] Boots, B.N. and Getis, A. (1998) Point pattern analysis. Sage Publications, Newbury Park.

[29] Cliff, A.C. and Ord, J.K. (1973) Spatial autocorrelation. Pion Limited, London.

[30] Grubesic, T.H. (2008) Zip codes and spatial analysis: Problems and prospects. Socio-Economic Planning Sciences, 42, 129-149. doi:10.1016/j.seps.2006.09.001

[31] Cliff, A.D. and Ord, J.K. (1981) Spatial processes: Models and applications. Pion Limited, London.

[32] Ord, J.K. and Getis, A. (1995) Local spatial autocorrelation statistics: Distributional issues and an application. Geographical Analysis, 27, 286-306. doi:10.1111/j.1538-4632.1995.tb00912.x

[33] Tobler, W. (1979) Cellular geography. In: Gale, S. and Olsson, G., Eds., Philosophy in Geography, Riedel, Dordrecht, 379-386.

[34] Moore, D.A. and Carpenter, T.E. (1999) Spatial analytical methods and geographic information systems: Use in health research and epidemiology. Epidemiologic $R e$ views, 21, 143-161.
[35] Griffith, D.A. and Arnrhein, C.G. (1991) Statistical analysis for geographers. Prentice Hall, Englewood Cliffs.

[36] Kitron, U. and Kazmierczak, J.J. (1997) Spatial analysis of the distribution of Lyme disease in Wisconsin. American Journal of Epidemiology, 145, 558-566.

[37] Manley, D., Flowerdew, R. and Steel, D. (2006) Scales, levels and processes: Studying spatial patterns of British census variables. Computers, Environment and Urban Systems, 30, 143-160. doi:10.1016/j.compenvurbsys.2005.08.005

[38] Matisziw, T.C., Grubesic, T.H. and Wei, H. (2008) Downscaling spatial structure for the analysis of epidemiological data. Computers, Environment and Urban Systems, 32, 81-93.

[39] De Smith, M.J., Goodchild, M.F. and Longley, P.A. (2007) Geospatial Analysis: A comprehensive guide to principles, techniques and software tools. Matador, Leicester.

[40] Lin, J.T., Wang, L.Y., Wang, J.T., Wang, T.H. and Chen, C.J. (1995) Ecological study of association between Helicobacter pylori infection and gastric cancer in Taiwan. Digestive Diseases and Sciences, 40, 385-388. doi:10.1007/BF02065425

[41] Yang, Y.H., Lee, H.Y., Tnug, S. and Shieh, T.Y. (2001) Epidemiological survey of oral submucous fibrosis and leukoplakia in aborigines of Taiwan. Journal of Oral Pathology \& Medicine, 30, 213-219. doi:10.1034/j.1600-0714.2001.300404.X

[42] Chiang, C.T., Hwang, Y.H., Su, C.C., Tsai, K.Y., Lian, I.B., Yuan, T.H. and Chang, T.K. (2010) Elucidating the underlying causes of oral cancer through spatial clustering in high-risk areas of Taiwan with a distinct gender ratio of incidence. Geospatial Health, 4, 231-242.

[43] Huang, J.Q., Sridhar, S., Chen, Y. and Hunt, R.H. (1998) Meta-analysis of the relationship between Helicobacter pylori seropositivity and gastric cancer. Gastroenterology, 114, 1169-1179. doi:10.1016/S0016-5085(98)70422-6

[44] Eslick, G.D., Lim, L.L. and Byles, J. (1999) Association of Helicobacter pylori infection with gastric carcinoma: A meta-analysis. The American Journal of Gastroenterology, 94, 2373-2379. doi:10.1111/j.1572-0241.1999.01360.X

[45] Xue, F.B., Xu, Y.Y. and Wan, Y. (2001) Association of Helicobacter pylori infection with gastric carcinoma: A meta-analysis. World Journal of Gastroenterology, 7, 801-804.

[46] Wang, C., Yuan, Y. and Hunt, R.H. (2007) The association between Helicobacter pylori infection and early gastric cancer: A meta-analysis. World Journal of Gastroenterology, 102, 1789-1798. doi:10.1111/j.1572-0241.2007.01335.x

[47] Teh, B.H., Lin, J.T., Pan, W.H., Lin, S.H., Wang, L.Y., Lee, T.K. and Chen, C.J. (1994) Seroprevalence and associated risk factors of Helicobacter pylori infection in Taiwan. Anticancer Research, 14, 1389-1392. 